Article

\title{
Effects of Chirality on the Antifungal Potency of Methylated Succinimides Obtained by Aspergillus fumigatus Biotransformations. Comparison with Racemic Ones
}

\author{
Maximiliano Sortino, Agustina Postigo and Susana Zacchino * \\ Pharmacognosy Area, Faculty of Biochemical and Pharmaceutical Sciences, \\ National University of Rosario, Suipacha 531, 2000-Rosario, Argentina; \\ E-Mails: msortino@fbioyf.unr.edu.ar (M.S.); aguspostigo@hotmail.com (A.P.) \\ * Author to whom correspondence should be addressed; E-Mail: szaabgil@citynet.net.ar; \\ Tel./Fax: +54-341-437-5315.
}

Received: 24 April 2013; in revised form: 13 May 2013 / Accepted: 13 May 2013 /

Published: 15 May 2013

\begin{abstract}
Eighteen $(3 R)$ and $(3 R, 4 R)-N$-phenyl-, $N$-phenylalkyl and $N$-arylsuccinimides were prepared with high enantioselectivity by biotransformation of maleimides with A. fumigatus. This environmentally friendly, clean and economical procedure was performed by the whole-cell fungal bioconversion methodology. Their corresponding eighteen racemic succinimides were prepared instead by synthetic methods. Both, the racemic and the chiral succinimides were tested simultaneously by the microbroth dilution method of CLSI against a panel of human opportunistic pathogenic fungi of clinical importance. Chiral succinimides showed higher antifungal activity than the corresponding racemic ones and the differences in activity were established by statistical methods. The bottlenecks for developing chiral drugs are how to obtain them through a low-cost procedure and with high enantiomeric excess. Results presented here accomplish both these objectives, opening an avenue for the development of asymmetric succinimides as new antifungal drugs for pharmaceutical use.
\end{abstract}

Keywords: biotransformation; Aspergillus fumigatus; enantioselective reduction; enhanced antifungal activity; chiral succinimides; methylated succinimides 


\section{Introduction}

Chiral succinimides, containing asymmetric carbons (in position 3- or 3,4- of the imido ring) have demonstrated to be core structural units with interesting biological activities. They have shown anxiolytic, antidepressant effects, and the ability to inhibit protein synthesis and human enzymes, such as leucocyte elastase, cathepsin G, proteinase 3 and glycosidase, among others. As a consequence, they have become good clinical drug candidates for several diseases [1-6].

Regarding antimicrobial activity, the chiral succinimides andrimid and moraimide B showed potent in vitro antibacterial activity against antibiotic-resistant human pathogens as methicillin-resistant Staphylococcus aureus [7]. In turn, hirsutellones inhibited the growth of Mycobacterium tuberculosis [4]. These findings have led to an increased interest in the clinical use of these asymmetric structures as a class of potential antimicrobial agents.

The bottleneck for developing chiral drugs for pharmaceutical use is to obtain them in a cheap and friendly procedure with high enantiomeric excesses (ees). Asymmetric synthesis by chemical procedures typically requires the use of expensive catalysts containing transition metal ions [8], which often prevents its commercial development. In contrast, the application of biocatalysts using whole cells in their native forms in aqueous/organic media has shown to be a highly selective, environmentally safe and cost effective method of producing enantiomeric compounds [9-12].

In the course of our project aimed at generating new chiral compounds through fungal biotransformations, we previously reported the preparation of $(3 R)-(+)$-methyl- $N$-phenylsuccinimide (1a) and $(3 R, 4 R)-(+)$-dimethyl- $N$-phenylsuccinimide (2a). These compounds were obtained in $>99 \%$ ee by bioconversion of 3-methyl- $N$-phenyl- and 3,4-dimethyl- $N$-phenylmaleimide with Aspergillus fumigatus ATCC 26934, which proved to be the most effective catalyst among the fifteen strains tested [13]. In a subsequent paper, we reported the production of $(3 R)-(+)$-methyl- $N$-phenylalkylsuccinimides $\mathbf{1 b}-\mathbf{e}$ and $(3 R, 4 R)-(+)$-dimethyl- $N$-phenylalkylsuccinimides $\mathbf{2 b}-\mathbf{e}$ [alkyl chain $=\left(\mathrm{CH}_{2}\right)_{\mathrm{n}}(\mathrm{n}=1-4)$ ] (Figure 1) with excellent enantioselectivities $(>99 \%$ ee $)$ from 3-methyl- and 3,4-dimethyl- $N$-phenylalkylmaleimides, with the same A. fumigatus strain [14].

Figure 1. Structures of $(3 R)-(+)$-methyl- $N$-phenylsuccinimide 1a, $(3 R, 4 R)-(+)$-dimethyl- $N$ phenylsuccinimide 2a, $(3 R)-(+)$-methyl- $N$-phenylalkylsuccinimides $\mathbf{1 b}-\mathbf{e}$ and $(3 R, 4 R)-(+)-$ dimethyl- $N$-phenylalkylsuccinimides $\mathbf{2 b}-\mathbf{e}$ [alkyl chain $\left.=\left(\mathrm{CH}_{2}\right)_{\mathrm{n}}(\mathrm{n}=1-4)\right]$.
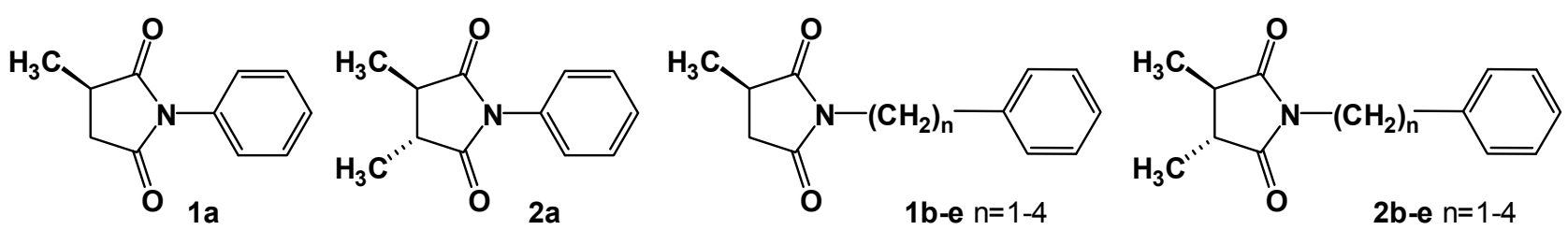

This prompted us to expand the knowledge on the ability of A. fumigatus to stereoselectively hydrogenate eight related 3-methyl- $N$-arylmaleimides to produce asymmetric succinimides 3-10 (Scheme 1). These compounds possess a substituted benzene ring with either electron-withdrawing or electron-donor groups on its $p$ - or $o$-positions. It is known that the application of the same microorganism on different substrates does not always result in similar transformations or enantioselectivities of catalyzed reactions. 
Scheme 1. Biotransformation of 3-methyl- $N$-arylmaleimides 11-18 to $(3 R)-(+)$-methyl- $N$ arylsuccinimide 3-10 with Aspergillus fumigatus ATCC 26934.

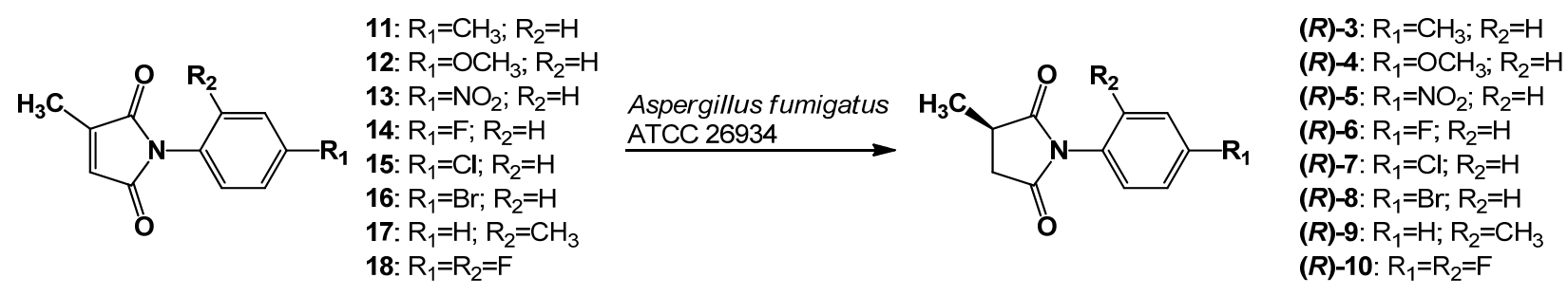

Chiral compounds 3-10, along with the previously obtained chiral $\mathbf{1 a}-\mathbf{e}$ and $\mathbf{2 a}-\mathbf{e}$, were tested here for antifungal properties against a panel of human opportunistic pathogenic fungi using standardized procedures. In addition, racemic succinimides $( \pm)-\mathbf{- 1 a}-\mathbf{e} ; \mathbf{- 2 a}-\mathbf{e} ; \mathbf{- 3}-\mathbf{1 0}$ were synthesized and tested simultaneously against the same panel of fungi in order to compare their antifungal activities. It is well known that isomers can differ in their biological activities, thus the knowledge of the properties of both, racemic and enantiomeric forms of a compound is of great significance in the pharmaceutical field, leading to a better understanding of the concentration-effect relationships, adverse effects, activity or toxicity [15].

\section{Results and Discussion}

For the sake of simplicity, the thirty six compounds: racemic ( $r a c)-$ and chiral 1a-e, 2a-e, 3-10, were grouped into three classes: (A) (rac)- and (3R)-methyl- $N$-phenyl- and $N$-phenylalkyl-succinimides 1a-1e; (B) (rac)- and (3R,4R)-dimethyl- $N$-phenyl- and $N$-phenylalkyl- succinimides $\mathbf{2 a - 2 e}$; and (C) (rac)- and (3R)-methyl- $N$-arylsuccinimides 3-10.

As stated above, $(3 R)$ - and $(3 R, 4 R)$-succinimides of groups (A) and (B) (compounds 1a-e and $\mathbf{2 a}-\mathbf{e})$ were previously obtained with $>99 \%$ ee by fungal biotransformation of the respective maleimides with A. fumigatus ATCC 26934 [13,14,16]. In turn, racemic ones were obtained by catalytic hydrogenation of the respective maleimides, as previously reported [14].

Chiral compounds of group $\mathrm{C}[(3 R)-\mathbf{3}-\mathbf{1 0})]$, on the other hand, were obtained by submitting the respective maleimides 11-18 to biotransformation with the same strain of A. fumigatus used for obtaining chiral succinimides of groups (A) and (B) (Scheme 1). (Rac)-3-10 were obtained by catalytic hydrogenation with $\mathrm{H}_{2}(\mathrm{Pd} / \mathrm{C})$. In turn, maleimides 11-18 were obtained following reported procedures [14].

The structures of 3-10 were corroborated by MS and ${ }^{1} \mathrm{H}$ - and ${ }^{13} \mathrm{C}-\mathrm{NMR}$. To assist in the assignment of both the absolute configuration and the ee of each chiral succinimide, $R$-enantiomers of $\mathbf{3 - 1 0}$ were also synthesized from $(R)-2$-methylsuccinic acid 19 and the respective anilines 20-27 (Scheme 2) [17]. Synthetic (3R)-3-10 were all dextrorotatory, therefore indicating that the biotransformation products (+)-3-10 had the $R$-configuration.

Chiral GC of rac-and (3R)-(3-10) allowed us to determine that (3R)-enantiomers $\mathbf{3}-\mathbf{1 0}$ eluted before the $S$-ones. These data were used to determine the ee of each biotransformation mixture and to confirm its absolute configuration. Results showed that 11-18 were converted into $(3 R)-(+)-\mathbf{3}-\mathbf{1 0}$ with $e e \geq 98 \%$. Table 1 shows \% conversion; \% ee and absolute configurations of $\mathbf{3}-\mathbf{1 0}$ [data of succinimide $R$-1a, obtained from 3-methyl- $N$-phenylmaleimide (28) [13], were also included]. 
Scheme 2. Synthesis of (3R)-methyl- $N$-aryl-succinimides 3-10 from (2R)-methylsuccinic acid $\mathbf{1 9}$ and respective anilines $\mathbf{2 0 - 2 7 .}$

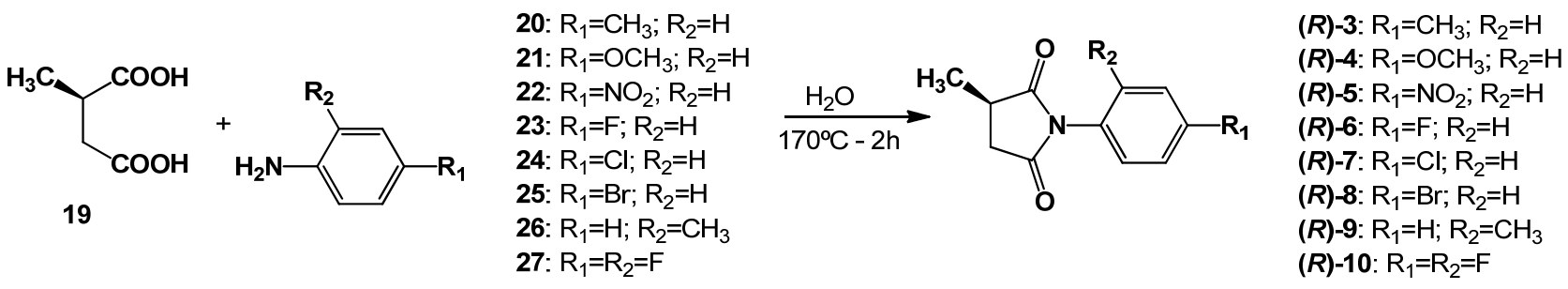

Table 1. Biotransformation of 3-methyl- $N$-arylmaleimides 11-18 to $(3 R)-(+)$-methyl- $N$ arylsuccinimides $\mathbf{3}-\mathbf{1 0}$.

\begin{tabular}{cccccc} 
& & & & \\
\hline
\end{tabular}

* Results previously reported [13]; \% ee: \% enantiomeric excess (calculated by chiral GC); \% Conv: Conversion percentages (determined by GC analysis by using the TIC (total ion current) with the following equation: \% conversion: product TIC/(product TIC + substrate TIC) $\times 100$.

The above results expand the knowledge on the ability of A. fumigatus ATCC 26934 to enantioselectively hydrogenate eight related prochiral 3-methyl-N-arylmaleimides 11-18 to produce chiral succinimides $(3 R)-\mathbf{3 - 1 0}$ with high enantioselectivity and in high yields. The fungus showed the same enantioface preference, irrespective of the substituent on the benzene ring which was the same results that has been observed with their analogues $\mathbf{1 a}-\mathbf{e}$ and $\mathbf{2 a}-\mathbf{e}[13,14]$. To our knowledge, there are no previous reports on chiral synthesis of (3R)-methyl- $N$-arylsuccinimides 3, 5-10 by any of the chemical or enzymatic methods. Instead, $(3 R)-(+)-4$ has been previously obtained by biotransformation with the plant Marchantia polymorpha [18].

Compounds (3R)-(3-10), along with the previously obtained chiral compounds $\mathbf{1 a - e}$ and $\mathbf{2 a - e}$, were tested for antifungal properties against a panel of eleven human opportunistic pathogenic fungi comprising yeasts (Candida spp., Cryptococcus neoformans, Saccharomyces cerevisiae and the dermatophytes Microsporum gypseum, Trichophyton rubrum and Trichophyton mentagrophytes). The selection of these species was due to their high clinical incidence mainly among immunocompromised patients. Thus, species of the genus Candida are among the leading causes of nosocomial, blood stream infections worldwide and, although $C$. albicans was in the past the usual species associated with 
invasive infections, at present non-albicans Candida spp. (C. tropicalis, C. glabrata, C. parapsilopsis, C. krusei and C. lusitaneae) comprise more than half of human candidiasis isolates [19].

In turn, C. neoformans was selected because it remains an important life-threatening species for immunocompromised hosts, particularly for patients infected with HIV and therefore, new compounds that act against this fungus are highly welcome [20,21]. Regarding dermatophytes of the genus Microsporum and Trichophyton, they were selected because both genera are the cause of approximately 80-93\% of chronic and recurrent human superficial infections which, although not life-threatening, diminish the quality of life of patients because they are difficult to eradicate [22].

To determine the Minimum Inhibitory Concentration (MIC), amounts of compounds from $250 \mu \mathrm{g} \cdot \mathrm{mL}^{-1}$ were incorporated into growth media according to the CLSI standardized procedures [23,24]. Amphotericin B, terbinafine, and ketoconazole were used as positive controls. The Enhancement Ratio (ER), which is a measure of how many-fold the MIC was reduced in each enantiomeric compound compared to its corresponding racemic one, was calculated as the ratio between MIC ( $r a c$ )/MIC (enantiomer). Results are shown in Table 2.

Table 2. Antifungal activity (MICs in $\mu \mathrm{g} \cdot \mathrm{mL}^{-1}$ ) of 3-methyl- $N$-phenyl- or $N$ phenylalkylsuccinimides 1a-e (A); 3,4-dimethyl- $N$-phenyl- or phenylalkylsuccinimides 2a-e (B); and 3-methyl- $N$-arylsuccinimides 3-10 (C), in their racemic and enantiomeric forms against a panel of yeasts and dermatophytes.

A

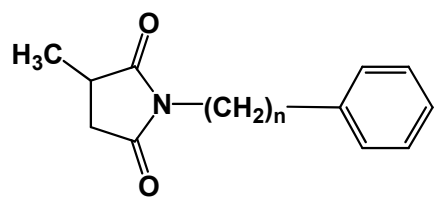

B

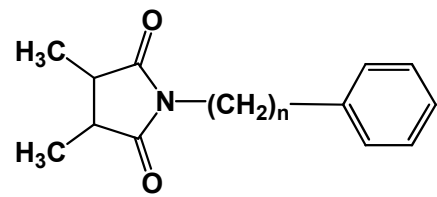

C

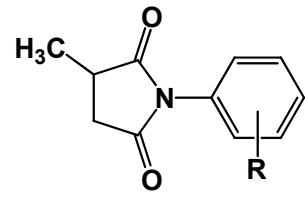

\begin{tabular}{|c|c|c|c|c|c|c|c|c|c|c|c|c|c|c|c|c|}
\hline & & Type & $\mathrm{n}$ & & Conf. & $\mathrm{Ca}^{1}$ & $C t^{2}$ & $C k^{3}$ & $\mathrm{Cg}^{4}$ & $C_{p}^{5}$ & $\mathrm{Cl}^{6}$ & $S c^{7}$ & $C n^{8}$ & $M^{9}$ & $\operatorname{Tr}^{10}$ & $T m^{11}$ \\
\hline & & & & & $R-$ & 62.5 & 62.5 & 62.5 & 62.5 & 62.5 & 62.5 & 62.5 & 62.5 & 62.5 & 31.3 & 62.5 \\
\hline & \multirow[t]{3}{*}{$1 \mathrm{a}$} & A & 0 & - & rac- & 125 & 125 & 125 & 125 & 125 & 125 & 125 & 125 & 62.5 & 125 & 62.5 \\
\hline & & & & & ER & 2 & 2 & 2 & 2 & 2 & 2 & 2 & 2 & - & 4 & - \\
\hline & & & & & $R-$ & 15.6 & 31.3 & 62.5 & 62.5 & 62.5 & 31.3 & 62.5 & 15.6 & 125 & 62.5 & 62.5 \\
\hline & \multirow[t]{3}{*}{$1 \mathrm{~b}$} & A & 1 & - & rac- & 62.5 & 125 & 125 & 125 & 125 & 125 & 125 & 62.5 & 250 & 125 & 250 \\
\hline & & & & & ER & 4 & 4 & 2 & 2 & 2 & 4 & 2 & 4 & 2 & 2 & 4 \\
\hline & & & & & $R-$ & 31.3 & 62.5 & 125 & 62.5 & 31.3 & 62.5 & 62.5 & 62.5 & 125 & 62.5 & 31.3 \\
\hline \multirow[t]{8}{*}{1} & $1 \mathrm{c}$ & A & 2 & 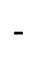 & rac- & 125 & 125 & 125 & 125 & 125 & 125 & 125 & 125 & 125 & 62.5 & 62.5 \\
\hline & & & & & ER & 4 & 2 & - & 2 & 4 & 2 & 2 & 2 & - & - & 2 \\
\hline & & & & & $R-$ & 62.5 & 125 & 125 & 62.5 & 125 & 125 & 125 & 62.5 & 62.5 & 125 & 62.5 \\
\hline & 1d & A & 3 & - & rac- & 125 & 125 & 125 & 125 & 125 & 125 & 125 & 125 & 125 & 250 & 250 \\
\hline & & & & & ER & 2 & - & - & - & - & - & - & 2 & 2 & 2 & 4 \\
\hline & & & & & $R-$ & 31.3 & 31.3 & 62.5 & 125 & 62.5 & 125 & 62.5 & 31.3 & 125 & 62.5 & 31.3 \\
\hline & $1 \mathrm{e}$ & A & 4 & - & rac- & 62.5 & 125 & 125 & 125 & 125 & 125 & 125 & 125 & 250 & 125 & 125 \\
\hline & & & & & ER & 2 & 4 & 2 & - & 2 & - & 2 & 4 & 2 & 2 & 4 \\
\hline
\end{tabular}


Table 2. Cont.

\begin{tabular}{|c|c|c|c|c|c|c|c|c|c|c|c|c|c|c|c|}
\hline & Type & n & & onf. & $C a^{1}$ & $C t^{2}$ & $C k^{3}$ & $C g^{4}$ & $C p^{5}$ & $\mathrm{Cl}^{6}$ & $S c^{7}$ & $C n^{8}$ & $M g^{9}$ & $T r^{10}$ & $T m^{11}$ \\
\hline \multirow{15}{*}{2} & \multirow{3}{*}{ B } & \multirow{3}{*}{0} & & $3 R, 4 R-$ & 31.3 & 31.3 & 62.5 & 62.5 & 31.3 & 62.5 & 62.5 & 62.5 & 125 & 125 & 62.5 \\
\hline & & & & rac- & 125 & 62.5 & 62.5 & 125 & 62.5 & 62.5 & 125 & 125 & 125 & 125 & 125 \\
\hline & & & & ER & 4 & 2 & - & 2 & 2 & - & 2 & 2 & - & - & 2 \\
\hline & \multirow{3}{*}{ B } & \multirow{3}{*}{1} & & $3 R, 4 I$ & 31.3 & 62.5 & 62.5 & 125 & 62.5 & 62.5 & 125 & 62.5 & 62.5 & 62.5 & 31.3 \\
\hline & & & & rac- & 125 & 125 & 125 & 125 & 125 & 125 & 125 & 125 & 62.5 & 62.5 & 125 \\
\hline & & & & ER & 4 & 2 & 2 & - & 2 & 2 & - & 2 & - & - & 4 \\
\hline & \multirow{3}{*}{ B } & \multirow{3}{*}{2} & & $3 R, 4 R-$ & 15.6 & 125 & 125 & 62.5 & 62.5 & 62.5 & 125 & 62.5 & 125 & 125 & 62.5 \\
\hline & & & & rac- & 125 & 125 & 250 & 125 & 250 & 125 & 125 & 125 & 250 & 250 & 62.5 \\
\hline & & & & ER & 8 & - & 2 & 2 & 4 & 2 & - & 2 & 2 & 2 & - \\
\hline & \multirow{3}{*}{ B } & \multirow{3}{*}{3} & & $3 R, 4 R-$ & 31.3 & 62.5 & 62.5 & 62.5 & 62.5 & 62.5 & 62.5 & 62.5 & 62.5 & 31.3 & 31.3 \\
\hline & & & & rac- & 62.5 & 125 & 125 & 125 & 125 & 250 & 125 & 125 & 125 & 62.5 & 125 \\
\hline & & & & ER & 2 & 2 & 2 & 2 & 2 & 4 & 2 & 2 & 2 & 2 & 4 \\
\hline & \multirow{3}{*}{$B$} & \multirow{3}{*}{4} & & $3 R, 4 R-$ & 62.5 & 62.5 & 62.5 & 62.5 & 62.5 & 62.5 & 62.5 & 31.3 & 62.5 & 62.5 & 62.5 \\
\hline & & & - & rac- & 125 & 125 & 62.5 & 125 & 125 & 125 & 125 & 125 & 125 & 125 & 125 \\
\hline & & & & ER & 2 & 2 & - & 2 & 2 & 2 & 2 & 4 & 2 & 2 & 2 \\
\hline & & & & $R-$ & 62.5 & 62.5 & 62.5 & 62.5 & 62.5 & 62.5 & 62.5 & 62.5 & 125 & 125 & 125 \\
\hline 3 & C & 0 & $4^{\prime}-\mathrm{CH}_{3}$ & rac- & 250 & 250 & 125 & 125 & 250 & 250 & 125 & 62.5 & 250 & 250 & 250 \\
\hline & & & & ER & 4 & 4 & 2 & 2 & 4 & 4 & 2 & - & 2 & 2 & 2 \\
\hline & & & & $R-$ & 125 & 62.5 & 62.5 & 62.5 & 62.5 & 62.5 & 125 & 62.5 & 125 & 125 & 125 \\
\hline 4 & $\mathrm{C}$ & 0 & 4'-OMe & rac- & 250 & 125 & 125 & 125 & 125 & 125 & 125 & 62.5 & 250 & 250 & 250 \\
\hline & & & & ER & 2 & 2 & 2 & 2 & 2 & 2 & - & - & 2 & 2 & 2 \\
\hline & & & & $R-$ & 62.5 & 125 & 62.5 & 125 & 62.5 & 125 & 125 & 62.5 & 125 & 125 & 125 \\
\hline 5 & $\mathrm{C}$ & 0 & $4^{\prime}-\mathrm{NO}_{2}$ & rac- & 250 & 125 & 125 & 125 & 125 & 125 & 125 & 125 & 250 & 250 & 250 \\
\hline & & & & ER & 4 & - & 2 & - & 2 & 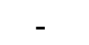 & 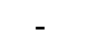 & 2 & 2 & 2 & 2 \\
\hline & & & & $R-$ & 62.5 & 62.5 & 31.3 & 62.5 & 31.3 & 62.5 & 62.5 & 62.5 & 62.5 & 62.5 & 62.5 \\
\hline 6 & $\mathrm{C}$ & 0 & 4'-F & $\overline{r a c-}$ & 250 & 250 & 125 & 125 & 250 & 250 & 125 & 62.5 & 125 & 125 & 125 \\
\hline & & & & ER & 4 & 4 & 4 & 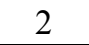 & 8 & 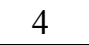 & 2 & - & 2 & 2 & 2 \\
\hline & & & & $R-$ & 125 & 62.5 & 125 & 62.5 & 125 & 62.5 & 62.5 & 31.3 & 125 & 125 & 125 \\
\hline 7 & $\mathrm{C}$ & 0 & $4^{\prime}-\mathrm{Cl}$ & rac- & 250 & 250 & 125 & 250 & 250 & 250 & 125 & 125 & 250 & 250 & 250 \\
\hline & & & & ER & 2 & 4 & - & 4 & 2 & 4 & 2 & 4 & 2 & 2 & 2 \\
\hline & & & & $\underline{R-}$ & 125 & 62.5 & 62.5 & 62.5 & 62.5 & 62.5 & 62.5 & 62.5 & 62.5 & 125 & 125 \\
\hline 8 & $\mathrm{C}$ & 0 & $4^{\prime}-\mathrm{Br}$ & rac- & 250 & 250 & 125 & 125 & 250 & 250 & 125 & 125 & 125 & 250 & 125 \\
\hline & & & & ER & 2 & 4 & 2 & ? & 4 & 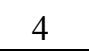 & 2 & 2 & 2 & 2 & - \\
\hline & & & & $R-$ & 125 & 125 & 62.5 & 125 & 125 & 125 & 125 & 62.5 & 125 & 125 & 125 \\
\hline 9 & $\mathrm{C}$ & 0 & $2^{\prime}-\mathrm{CH}_{3}$ & rac- & 250 & 125 & 125 & 125 & 250 & 125 & 125 & 125 & 250 & 250 & 250 \\
\hline & & & & ER & 2 & - & 2 & - & 2 & - & - & 2 & 2 & 2 & 2 \\
\hline & & & & $R-$ & 62.5 & 62.5 & 31.3 & 62.5 & 31.3 & 125 & 62.5 & 62.5 & 62.5 & 62.5 & 125 \\
\hline 10 & $\mathrm{C}$ & 0 & $2^{\prime}, 4^{\prime}-F_{2}$ & rac- & 250 & 250 & 125 & 125 & 250 & 250 & 125 & 125 & 125 & 250 & 250 \\
\hline & & & & ER & 4 & 4 & 4 & 2 & 8 & 2 & 2 & 2 & 2 & 4 & 2 \\
\hline Amphot & $\operatorname{cin} B$ & & & & 0.97 & 0.48 & 0.49 & 0.49 & 0.98 & 0.98 & 0.48 & 0.24 & 0.12 & 0.06 & 0.06 \\
\hline etocona & zole & & & & 0.48 & 0.12 & 62.5 & 1.95 & 0.98 & 0.98 & 0.48 & 0.24 & 0.06 & 0.03 & 0.03 \\
\hline rbina & & & & & - & - & - & - & - & - & - & - & 0.03 & 0.01 & 0.03 \\
\hline
\end{tabular}

1: Candida albicans ATCC 10231, 2: C. tropicalis CCC 131-2000: C. krusei CCC 117-2000, 4: C. glabrata CCC 115-2000, 5: C. parapsilosis CCC 124-2000, 6: C. lusitaniae CCC 134-2000, 7: Saccharomyces cerevisiae ATCC 9763, 8: Cryptococcus neoformans ATCC 32264, 9: Microsporum gypseum CCC 115-2000, 10: Trichophyton rubrum CCC 113 2000, 11: T. mentagrophytes ATCC 9972. CCC = Colección de Cultivos del CEREMIC (Centro de Referencia en Micología). ATCC: American Type Culture Collection. Conf: configuration; $\mathrm{ER}=\mathrm{MIC}(\mathrm{rac}) / \mathrm{MIC}$ ( $R$ form) . 
The comparison of the 198 MICs of racemic mixtures (18 compounds x 11 fungi) with the same number of MICs of enantiomers, allowed us to detect that 158 MICs of chiral forms (80\%) were statistically significantly lower $(p<0.05)$ than the corresponding racemic form ones. Of them, 117 MICs were two orders of magnitude lower $(E R=2) ; 38$ MICs were four-fold lower $(E R=4)$, and three MICs were eight-fold lower $(\mathrm{ER}=8)$. C. albicans was shown to be the most sensitive species to chirality, showing enhancements in all pairs of compounds tested. The statistical analyses were performed by the non-parametric ANOVA, Kruskal-Wallis test followed by Dunn's multiple comparisons and Wilcoxon's signed rank test $(p<0.05)$.

This overall trend of a better antifungal behavior of $(3 R)$ - and $(3 R, 4 R)$ - forms, with respect to racemic ones against the whole panel of fungi, was analyzed within each group of compounds for each type of fungus (yeasts and dermatophytes) (Figure 2). Each graph shows the percentage of MICs of each value, calculated as follows: [number of MICs of each value $(250,125,62.5,31.3$ and $\left.15.6 \mu \mathrm{g} \cdot \mathrm{mL}^{-1}\right) \times 100 /$ total number of MICs] displayed by enantiomeric forms and by racemic mixtures within each group of compounds. The associations between MIC values and enantiomeric or racemic forms within each group and type of fungi for the different groups were established with the Score's test $(p<0.05)$ [25].

Figure 2. Percentages of the different MIC values (number of MICs at each concentration/total number of $\mathrm{MICs}) \times 100$, for the enantiomeric $(3 R)$ or $(3 R, 4 R)$ and racemic forms of succinimides of either group (A), (B), or (C) acting against yeasts and dermatophytes.
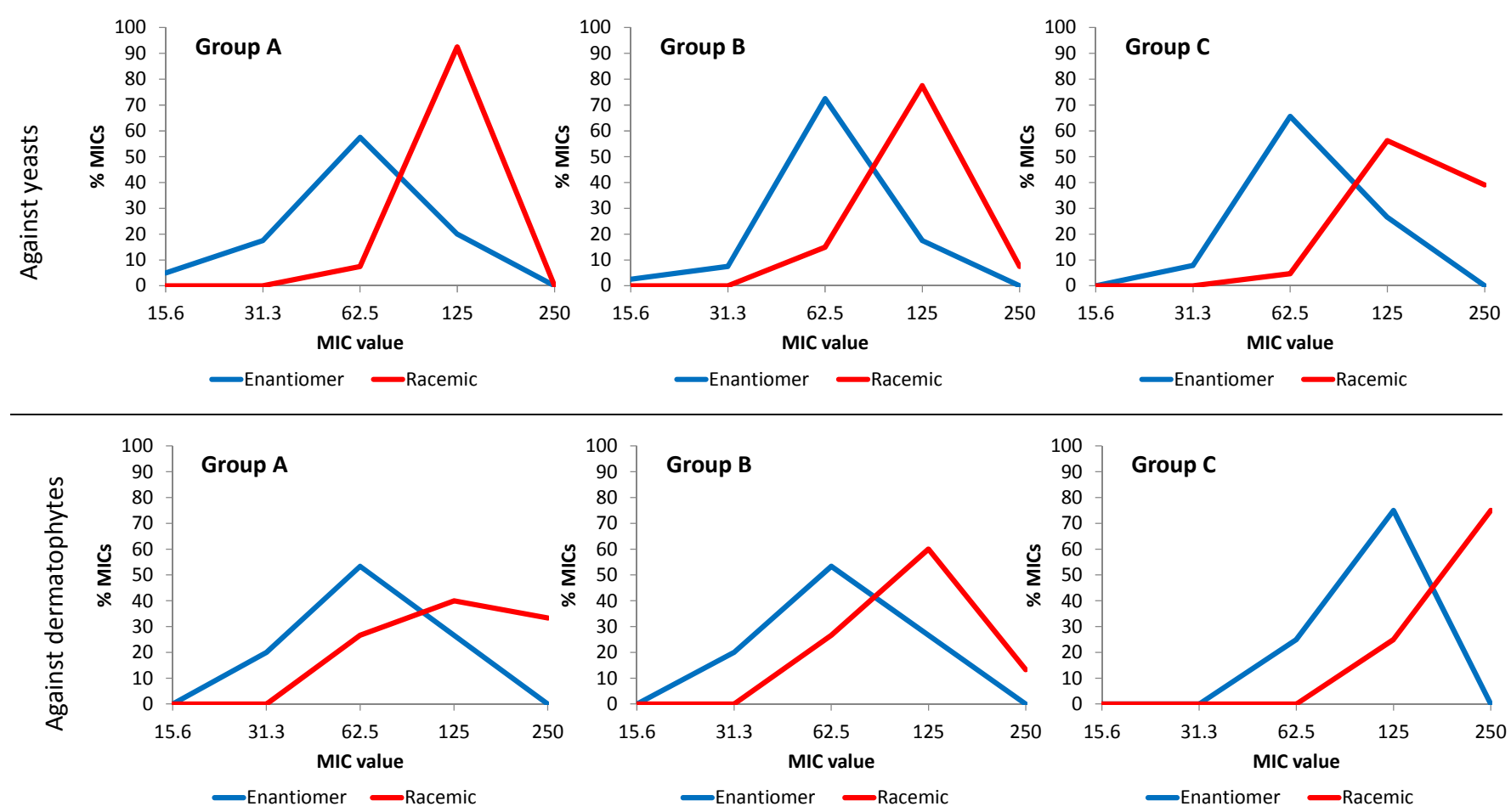

To corroborate the above findings from another point of view, we compared the percentages of fungal growth inhibition for the enantiomeric form and the racemic mixture of each compound, at a fixed concentration. Figure 3 shows the comparative antifungal inhibitory activities of chiral $v s$ racemic forms of each of the eighteen structures tested at $125 \mu \mathrm{g} \cdot \mathrm{mL}^{-1}$ as a measure of $\%$ of growth of C. albicans. 
Figure 3. Comparative values of the inhibitory activities of antifungal succinimides in their enantiomeric and racemic forms (at $125 \mu \mathrm{g} / \mathrm{mL}$ ) expressed as percentage of growth against Candida albicans ATCC 10231. * $p<0.05$.

a)

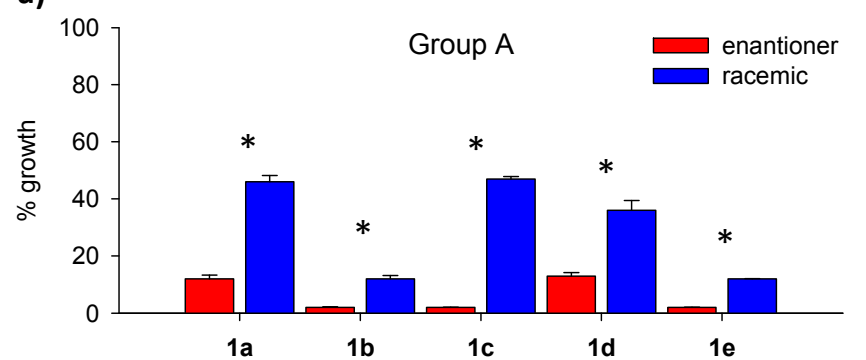

b)

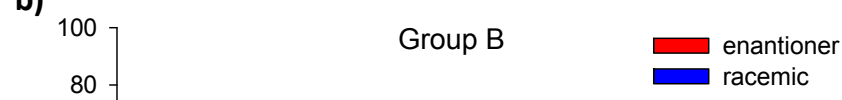

c)
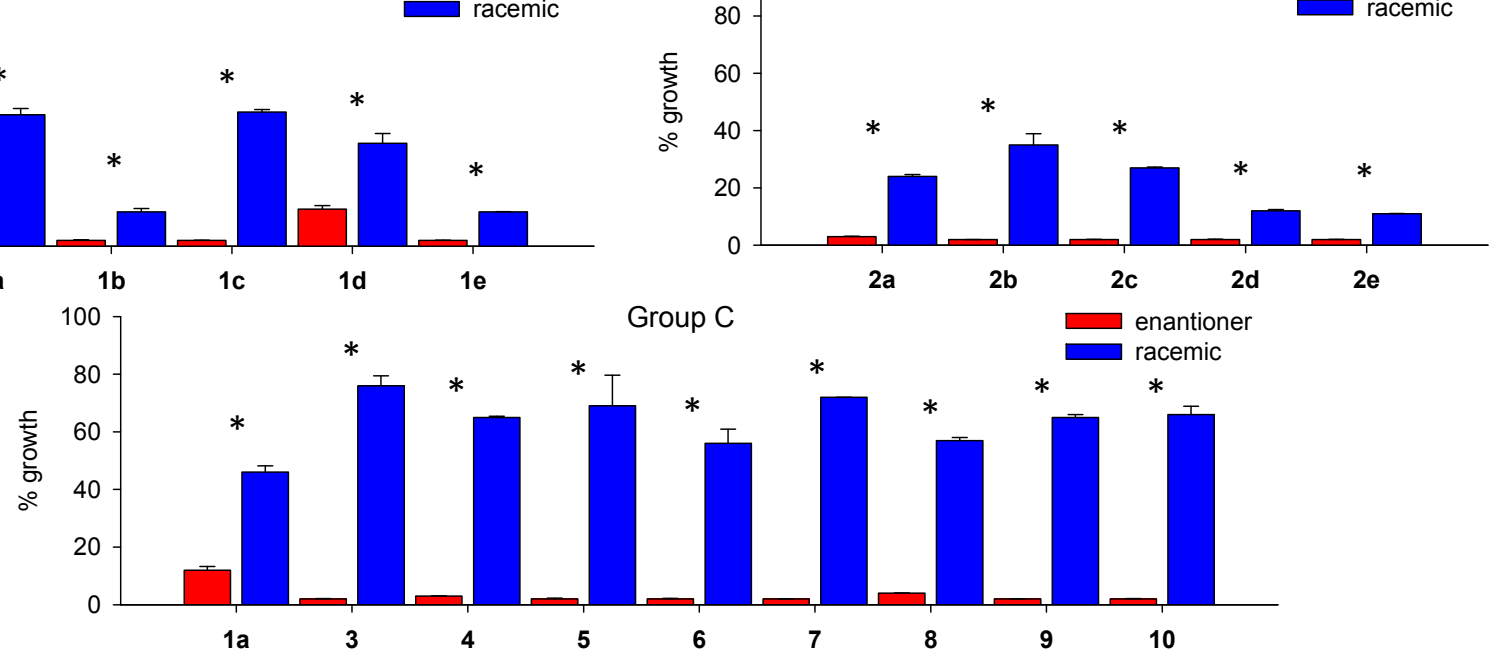

Group C

$2 c \quad 2 d$

It can be observed in the three groups, that each enantiomer showed a significantly lower percent of growth that its respective racemic form, confirming the previous analyses. These comparisons between groups were performed with Student's $t$ test.

It is worth noting that the overall antifungal behavior of chiral forms was better than that of racemic ones, irrespective of the succinimides' $N$-substituents, since chiral succinimides with or without an alkyl chain between the $N$ atom and the phenyl group, or with or without substituents in the $2^{\prime}$ and 4 '-positions of the benzene ring, showed better antifungal activity than their corresponding racemic forms.

\section{Experimental}

\subsection{General}

Solvents and reagents were purchased from Sigma-Aldrich (St. Louis, MO, USA) and were purified in the usual manner. ${ }^{1} \mathrm{H}$ - and ${ }^{13} \mathrm{C}$-NMR spectra were recorded on a Bruker (Karlsruhe, Germany) 300 MHz NMR spectrometer. Compounds were dissolved in deuterated solvents from commercial sources (Sigma-Aldrich) with tetramethylsilane (TMS) as the internal standard. Chemical shifts $(\delta)$ are reported in ppm relative to the solvent peak $\left(\mathrm{CHCl}_{3}\right.$ in $\mathrm{CDCl}_{3}$ at $7.26 \mathrm{ppm}$ for protons and at $77.0 \mathrm{ppm}$ for carbons). Signals are designated as follows: s, singlet; d, doublet; dd, doublets of doublets; $t$, triplet; m, multiplet; q, quartet. Melting points were obtained on or using an Electrothermal apparatus (Southend-on-Sea, Essex, UK) and are uncorrected. Optical rotations were measured with a Jasco DIB-1000 (Easton, USA) at room temperature. GC analyses were performed on a CG-MS Turbo Mass Perkin Elmer (Waltman, MA, USA), equipped with a fused silica gel column (SE-30 $25 \mathrm{~m} \times 0.22 \mathrm{~mm}$ I.D.) with $\mathrm{He}$ as a carrier gas in a column PE1 $30 \mathrm{~m} \times 0.25 \mathrm{~mm}$ (I.D.), film $0.1 \mu \mathrm{m}$, ionization energy $70 \mathrm{eV}$ with a temperature program of $70-200{ }^{\circ} \mathrm{C}$ at $10{ }^{\circ} \mathrm{C} \mathrm{min}{ }^{-1}$; total time $30 \mathrm{~min}$. Chiral gas chromatograms were obtained with a CG-MS QP2010-Plus (Shimadzu, Kioto, Japan), with He as a 
carrier gas on a Beta Dex-325 column (30 m $\times 0.25 \mathrm{~mm}$ I.D.), ionization energy $70 \mathrm{eV}$, with a temperature program of $60-200{ }^{\circ} \mathrm{C}$ at $2{ }^{\circ} \mathrm{C} \cdot \mathrm{min}^{-1}$; total time $80 \mathrm{~min}$.

\subsection{Synthesis}

3-Methyl-N-arylmaleimides 11-18. The synthesis of maleimides 11-18 was performed by mixing an equimolecular amount of substituted anilines 20-27 (5 mmol), dissolved in $\mathrm{CHCl}_{3}(1 \mathrm{~mL})$, and maleic anhydride 29 in $\mathrm{CHCl}_{3}(5 \mathrm{~mL})$ and stirring during $1 \mathrm{~h}$. The solid which precipitated out of the reaction mixture (maleamic acid) was filtered off. The whole amount of maleamic acid was dissolved in acetic anhydride $(5 \mathrm{~mL})$ and sodium acetate $(100 \mathrm{mg})$ was added. The mixture was heated for $2 \mathrm{~h}$ under reflux. The reaction was cooled, quenched with water and the aqueous solution was extracted with $\mathrm{Et}_{2} \mathrm{O}$, dried over $\mathrm{Na}_{2} \mathrm{SO}_{4}$, filtered, and the solvent evaporated. The product was purified by silica gel column chromatography using a mixture of hexane and ethyl acetate (9:1) as eluent. Spectroscopic NMR data of compound $\mathbf{1 2}$ was identical to that previously reported [18]. Compounds 11, 13-18 have been previously reported by Chemical Abstract Service (CAS) (11: CAS 3120-12-5, 13: CAS 10490-21-8, 14: CAS 883033-95-2, 15: CAS 59648-09-8, 16: CAS 134939-24-5, 17: CAS 131406-19-4, 18: CAS 124704-71-8) but, to the best of our knowledge, complete NMR spectral data were not available in the literature, so below, the complete spectroscopic data of these compounds are described.

3-Methyl-N-(4'-methylphenyl)maleimide (11). White solid. Mp: 92-95 ${ }^{\circ} \mathrm{C}$. Yield: $73 \%$. ${ }^{1} \mathrm{H}-\mathrm{NMR}$ $\left(\mathrm{CDCl}_{3}\right): 2.17\left(3 \mathrm{H}, \mathrm{d}, J=1.8 \mathrm{~Hz}, \mathrm{CH}_{3}\right) ; 2.37\left(3 \mathrm{H}, \mathrm{s}, \mathrm{CH}_{3}\right) ; 6.46(1 \mathrm{H}, \mathrm{q}, J=1.8 \mathrm{~Hz}, \mathrm{H}-3) ; 7.13-7.33$ (4H, m, HAr) ppm. ${ }^{13} \mathrm{C}-\mathrm{NMR}\left(\mathrm{CDCl}_{3}\right): 11.2 ; 21.1 ; 125.9 ; 127.4 ; 128.9 ; 129.7 ; 137.8 ; 145.7 ; 169.8$; 170.8 ppm. MS: $m / z 201\left(\mathrm{M}^{+}, 100 \%\right), 202\left(\mathrm{M}^{+}+1,13 \%\right)$.

3-Methyl-N-(4'-methoxyphenyl)maleimide (12). White solid. Mp: $99-101{ }^{\circ} \mathrm{C}$. Yield: $62 \%$. ${ }^{1} \mathrm{H}-\mathrm{NMR}$ $\left(\mathrm{CDCl}_{3}\right): \delta 2.17\left(3 \mathrm{H}, \mathrm{d}, J=1.8 \mathrm{~Hz}, \mathrm{CH}_{3}\right) ; 3.83\left(3 \mathrm{H}, \mathrm{s}, \mathrm{OCH}_{3}\right) ; 6.46(1 \mathrm{H}, \mathrm{q}, J=1.8 \mathrm{~Hz}, \mathrm{H}-3) ; 6.97(2 \mathrm{H}, \mathrm{d}$, $\left.J=8.9 \mathrm{~Hz}, \mathrm{H}-3^{\prime}, 5^{\prime}\right) ; 7.23\left(2 \mathrm{H}, \mathrm{d}, J=8.9 \mathrm{~Hz}, \mathrm{H}-2^{\prime} ; 6^{\prime}\right)$ ppm. ${ }^{13} \mathrm{C}-\mathrm{NMR}\left(\mathrm{CDCl}_{3}\right): 11.2 ; 55.5 ; 114.4$; $124.3 ; 127.4 ; 127.5 ; 145.7 ; 159.0 ; 169.9 ; 170.9$ ppm. MS: $m / z 217\left(\mathrm{M}^{+}, 100 \%\right), 218\left(\mathrm{M}^{+}+1,13 \%\right)$.

3-Methyl-N-(4'-nitrophenyl)maleimide (13). White solid. Mp: $126-128{ }^{\circ} \mathrm{C}$. Yield: $51 \%$. ${ }^{1} \mathrm{H}-\mathrm{NMR}$ $\left(\mathrm{CDCl}_{3}\right): 2.11\left(3 \mathrm{H}, \mathrm{d}, J=1.8 \mathrm{~Hz}, \mathrm{CH}_{3}\right) ; 6.48(1 \mathrm{H}, \mathrm{q}, J=1.8 \mathrm{~Hz}, \mathrm{H}-3) ; 7.62\left(2 \mathrm{H}, \mathrm{d}, J=9.3 \mathrm{~Hz}, \mathrm{H}-2^{\prime}, 6^{\prime}\right)$; $8.22\left(2 \mathrm{H}, \mathrm{d}, J=9.3 \mathrm{~Hz}, \mathrm{H}-3{ }^{\prime}, 5^{\prime}\right)$ ppm. ${ }^{13} \mathrm{C}-\mathrm{NMR}\left(\mathrm{CDCl}_{3}\right): 11.2 ; 124.4 ; 125.2 ; 127.9 ; 137.5 ; 146.0$; 146.4 ; 168.5 ; 169.7 ppm. MS: $m / z 232\left(\mathrm{M}^{+}, 100 \%\right), 233\left(\mathrm{M}^{+}+1,13 \%\right)$.

3-Methyl-N-(4'-fluorophenyl)maleimide (14). White solid. Mp: $122-125{ }^{\circ} \mathrm{C}$. Yield: $72 \%$. ${ }^{1} \mathrm{H}-\mathrm{NMR}$ $\left(\mathrm{CDCl}_{3}\right): 2.18\left(3 \mathrm{H}, \mathrm{d}, J=1.8 \mathrm{~Hz} ; \mathrm{CH}_{3}\right) ; 6.48(1 \mathrm{H}, \mathrm{q}, J=1.8 \mathrm{~Hz}, \mathrm{H}-3) ; 7.09-7.19$ (2H, m, H-2'; 6'); 7.28-7.36 (2H, m, H-3'; $\left.5^{\prime}\right)$ ppm. ${ }^{13} \mathrm{C}-\mathrm{NMR}\left(\mathrm{CDCl}_{3}\right)$ : 11.0; 115.8 (d, $\left.J=22.7 \mathrm{~Hz}\right) ; 127.1$ (d, $\left.J=8.9 \mathrm{~Hz}\right)$; 136.6; 139.8; 145.8; $161.4(\mathrm{~d}, J=248.5 \mathrm{~Hz}) ; 168.6 ; 169.5 \mathrm{ppm}$. MS: $m / z 205\left(\mathrm{M}^{+}, 100 \%\right)$, $206\left(\mathrm{M}^{+}+1,12 \%\right)$.

3-Methyl-N-(4'-chlorophenyl)maleimide (15). White solid. Mp: $127-130{ }^{\circ} \mathrm{C}$. Yield: $46 \%$. ${ }^{1} \mathrm{H}-\mathrm{NMR}$ $\left(\mathrm{CDCl}_{3}\right): 2.15\left(3 \mathrm{H}, \mathrm{d}, J=1.3 \mathrm{~Hz} ; \mathrm{CH}_{3}\right) ; 7.01(1 \mathrm{H}, \mathrm{d}, J=1.6 \mathrm{~Hz}, \mathrm{H}-3) ; 7.26\left(2 \mathrm{H}, \mathrm{d}, J=8.8 \mathrm{~Hz}, \mathrm{H}-3^{\prime}, 5^{\prime}\right)$; $7.44\left(2 \mathrm{H}, \mathrm{d}, J=8.7 \mathrm{~Hz}, \mathrm{H}-2^{\prime}, 6^{\prime}\right)$ ppm. ${ }^{13} \mathrm{C}-\mathrm{NMR}\left(\mathrm{CDCl}_{3}\right): 11.0 ; 126.2 ; 129.1 ; 136.4 ; 139.6 ; 142.3$; 150.0; 168.4; 180.7 ppm. MS: $m / z 221\left(\mathrm{M}^{+}, 100 \%\right) ; 223\left(\mathrm{M}^{+}+2,32 \%\right) ; 222\left(\mathrm{M}^{+}+1,12 \%\right)$. 
3-Methyl-N-(4'-bromophenyl)maleimide (16). White solid. Mp: $142-144{ }^{\circ} \mathrm{C}$. Yield: $56 \%$. ${ }^{1} \mathrm{H}-\mathrm{NMR}$ $\left(\mathrm{CDCl}_{3}\right): 2.17\left(3 \mathrm{H}, \mathrm{d}, J=1.8 \mathrm{~Hz}, \mathrm{CH}_{3}\right) ; 6.48(1 \mathrm{H}, \mathrm{q}, J=1.8 \mathrm{~Hz}, \mathrm{H}-3) ; 7.23-7.29(2 \mathrm{H}, \mathrm{d}, J=8.8 \mathrm{~Hz}$, $\left.3^{\prime}, 5^{\prime}-\mathrm{H}\right) ; 7.53-7.61\left(2 \mathrm{H}, \mathrm{d}, J=8.8 \mathrm{~Hz}, 2^{\prime}-6{ }^{\prime}-\mathrm{H}\right) \mathrm{ppm} .{ }^{13} \mathrm{C}-\mathrm{NMR}\left(\mathrm{CDCl}_{3}\right): 11.2 ; 121.2 ; 127.2 ; 127.6$; $130.4 ; 132.2 ; 145.9 ; 169.1 ; 170.6$ ppm. MS: $m / z 265\left(\mathrm{M}^{+}, 100 \%\right), 267\left(\mathrm{M}^{+}+2,97 \%\right), 265\left(\mathrm{M}^{+}+1\right.$, $12 \%), 268\left(\mathrm{M}^{+}+3,11 \%\right)$.

3-Methyl-N-(2'-methylphenyl)maleimide (17). White solid. Mp: $55-57{ }^{\circ} \mathrm{C}$. Yield: $52 \%$. ${ }^{1} \mathrm{H}-\mathrm{NMR}$ $\left(\mathrm{CDCl}_{3}\right): 2.15\left(3 \mathrm{H}, \mathrm{d}, J=1.8 \mathrm{~Hz}, \mathrm{CH}_{3}\right) ; 2.16\left(3 \mathrm{H}, \mathrm{s}, \mathrm{CH}_{3}\right) ; 6.47(1 \mathrm{H}, \mathrm{q}, J=1.8 \mathrm{~Hz}, \mathrm{H}-3) ; 7.10(1 \mathrm{H}, \mathrm{d}$, $\left.J=7.3, \mathrm{H}-3^{\prime}\right) ; 7.23-7.35$ (3H, m, H-4'-6') ppm. ${ }^{13} \mathrm{C}-\mathrm{NMR}\left(\mathrm{CDCl}_{3}\right): 11.1 ; 17.9 ; 126.8 ; 127.7 ; 128.7$; $129.5 ; 130.6 ; 131.1 ; 136.6 ; 146.2 ; 169.7 ; 170.7$ ppm. MS: $m / z 203\left(\mathrm{M}^{+}, 100 \%\right), 208\left(\mathrm{M}^{+}+1,13 \%\right)$.

3-Methyl-N-(2',4'-difluorophenyl)maleimide (18). White solid. Mp: 80-82 ${ }^{\circ} \mathrm{C}$. Yield: $85 \%$. ${ }^{1} \mathrm{H}-\mathrm{NMR}$ $\left(\mathrm{CDCl}_{3}\right): 2.01\left(3 \mathrm{H}, \mathrm{d}, J=1.8 \mathrm{~Hz}, \mathrm{CH}_{3}\right) ; 6.40(1 \mathrm{H}, \mathrm{d}, J=1.8 \mathrm{~Hz}, \mathrm{H}-3) ; 6.89$ (2H, q, $\left.J=9.3 \mathrm{~Hz} ; 5^{\prime}, 66^{\prime}-\mathrm{H}\right)$; $7.15\left(1 \mathrm{H}, \mathrm{dt}, J=8.6 \mathrm{~Hz} ; 3^{\prime}-\mathrm{H}\right) \mathrm{ppm} .{ }^{13} \mathrm{C}-\mathrm{NMR}\left(\mathrm{CDCl}_{3}\right): 10.8 ; 104.93(\mathrm{dd}, J=26.38,23.90 \mathrm{~Hz}) ; 111.78$ $(\mathrm{dd}, J=22.62,3.66 \mathrm{~Hz}) ; 115.66(\mathrm{dd}, J=13.43,3.89 \mathrm{~Hz}) ; 127.8 ; 130.8$ (dd, $J=10.09,1.35 \mathrm{~Hz}) ; 146.4$; $158.1(\mathrm{dd}, J=254.42,12.75 \mathrm{~Hz}) ; 162.5(\mathrm{dd}, J=250.8,11.3 \mathrm{~Hz}) ; 168.5 ; 169.7 \mathrm{ppm} . \mathrm{MS}: m / z 223$ $\left(\mathrm{M}^{+}, 100 \%\right), 224\left(\mathrm{M}^{+}+1,12 \%\right)$.

Rac-3-methyl-N-arylsuccinimides 3-10. Each maleimide 11-18 (2 mmol) was dissolved in $\mathrm{CH}_{2} \mathrm{Cl}_{2}$ ( $2 \mathrm{~mL}$ ) to which a catalytic amount of $5 \% \mathrm{Pd} / \mathrm{C}$ was added. Then, the mixture was exposed to a $\mathrm{H}_{2}$ atmosphere at room temperature for $2 \mathrm{~h}$. The crude mixture was filtered and the solvent was evaporated. The resulting product was purified by silica gel column chromatography using a mixture of hexane and ethyl acetate (9:1) as eluent.

(3R)-(+)-Methyl-N-arylsuccinimides 3-10. A mixture of $(R)-(+)$-methylsuccinic acid 19 (5.0 mmol in water) and substituted anilines 20-27 were maintained at $170{ }^{\circ} \mathrm{C}$ for $2 \mathrm{~h}$ and then cooled to $20^{\circ} \mathrm{C}$. The aqueous solution was extracted with $\mathrm{Et}_{2} \mathrm{O}$, dried over $\mathrm{Na}_{2} \mathrm{SO}_{4}$, filtered, and the solvent evaporated. The mixtures were subjected to silica gel column chromatography using a mixture of hexane and ethyl acetate (9:1) as eluent. Compounds 6, 9 and $\mathbf{1 0}$ ( $r a c$ or $R$ ) are not described in the literature. Spectroscopic NMR data of compound 4 was identical to that previously reported [18]. Compounds $r a c-3, r a c-5, r a c-7, r a c-8$ have been previously reported by Chemical Abstract Service (CAS) (3: CAS 105909-89-5, 5: CAS 33624-30-5, 7: CAS 25998-50-9, 8: CAS 134939-24-5) but, to the best of our knowledge, complete NMR spectral data were not available in the literature, so the complete spectroscopic data of these compounds are described below.

(3R)-(+)-Methyl-N-(4'-methylphenyl)succinimide (3), White solid. Mp: $113-115^{\circ} \mathrm{C}$. Yield: $58 \%$. ${ }^{1} \mathrm{H}-\mathrm{NMR}$ $\left(\mathrm{CDCl}_{3}\right): 1.45\left(3 \mathrm{H}, \mathrm{d}, J=7.2 \mathrm{~Hz}, \mathrm{CH}_{3}\right) ; 2.38\left(3 \mathrm{H}, \mathrm{s}, \mathrm{CH}_{3}\right) ; 2.49(1 \mathrm{H}, \mathrm{dd}, J=17.1 ; 3.8 \mathrm{~Hz}, \mathrm{H}-3 \mathrm{~b})$; 2.94-3.16 (2H, m, H-2;3a); 7.15 (2H, d, $\left.J=8.4 \mathrm{~Hz}, \mathrm{H}-2^{\prime} ; 6^{\prime}\right) ; 7.27$ (2H, d, $\left.J=8.5 \mathrm{~Hz}, \mathrm{H}-3^{\prime} ; 5^{\prime}\right)$ ppm. ${ }^{13} \mathrm{C}-\mathrm{NMR}\left(\mathrm{CDCl}_{3}\right)$ : 16.9; 21.2; 126.2; 129.3; 129.8; 138.7; 175.6; 179.7 ppm. MS: $\mathrm{m} / z 203\left(\mathrm{M}^{+}\right.$, $100 \%), 204\left(\mathrm{M}^{+}+1,13 \%\right)$. $[\alpha]_{\mathrm{D}}^{27}+5.2 \pm 1.2\left(\mathrm{c} 0.76, \mathrm{CHCl}_{3}\right)$.

(3R)-(+)-Methyl -N-(4'-methoxyphenyl)succinimide (4). White solid. Mp: $110-112{ }^{\circ} \mathrm{C}$. Yield: $65 \%$. ${ }^{1} \mathrm{H}-\mathrm{NMR}\left(\mathrm{CDCl}_{3}\right): 1.44\left(3 \mathrm{H}, \mathrm{d}, J=7.2 \mathrm{~Hz}, \mathrm{CH}_{3}\right) ; 2.48(1 \mathrm{H}, \mathrm{dd}, J=17.1 ; 3.8 \mathrm{~Hz}, \mathrm{H}-3 \mathrm{~b}) ; 2.94-3.15$ 
(2H, m, H-2 and H-3a); $3.82\left(3 \mathrm{H}, \mathrm{s}, \mathrm{OCH}_{3}\right) ; 6.98\left(2 \mathrm{H}, \mathrm{d}, J=8.4 \mathrm{~Hz}, \mathrm{H}-2^{\prime} ; 6^{\prime}\right) ; 7.19$ (2H, d, $J=8.5 \mathrm{~Hz}$, $\left.\mathrm{H}-3{ }^{\prime} ; 5^{\prime}\right)$ ppm. ${ }^{13} \mathrm{C}-\mathrm{NMR}\left(\mathrm{CDCl}_{3}\right): 16.9 ; 34.8 ; 36.6 ; 55.5 ; 114.5 ; 124.6 ; 127.6 ; 159.5 ; 175.7 ; 179.8$ ppm. MS: $m / z 219\left(\mathrm{M}^{+}, 100 \%\right), 220\left(\mathrm{M}^{+}+1,13 \%\right) .[\alpha]_{\mathrm{D}}^{27}+4.2 \pm 1.1\left(\mathrm{c} 0.96, \mathrm{CHCl}_{3}\right)$

(3R)-(+)-Methyl-N-(4'-nitrophenyl)succinimide (5. White solid. Mp: $111-112{ }^{\circ} \mathrm{C}$. Yield: $72 \% .{ }^{1} \mathrm{H}-\mathrm{NMR}$ $\left(\mathrm{CDCl}_{3}\right): 1.46\left(3 \mathrm{H}, \mathrm{d}, J=7.2 \mathrm{~Hz}, \mathrm{CH}_{3}\right) ; 2.56(1 \mathrm{H}, \mathrm{dd}, J=17.1 ; 3.9 \mathrm{~Hz}, \mathrm{H}-3 \mathrm{~b}) ; 2.98-3.18$ (2H, m, H-2; 3a); $7.61\left(2 \mathrm{H}, \mathrm{d}, J=9.3 \mathrm{~Hz}, \mathrm{H}-2^{\prime}, 6^{\prime}\right) ; 8.34\left(2 \mathrm{H}, \mathrm{d}, J=9.4 \mathrm{~Hz}, \mathrm{H}-3^{\prime}, 5^{\prime}\right)$ ppm. ${ }^{13} \mathrm{C}-\mathrm{NMR}\left(\mathrm{CDCl}_{3}\right)$ : 16.9 ; $34.9 ; 36.6 ; 123.1 ; 124.4 ; 126.8 ; 137.5 ; 174.5 ; 178.6$; ppm. MS: $m / z 234\left(\mathrm{M}^{+}, 100 \%\right), 235\left(\mathrm{M}^{+}+1\right.$, $12 \%) \cdot[\alpha]_{\mathrm{D}}^{27}+6.1 \pm 0.9\left(\mathrm{c} 0.86, \mathrm{CHCl}_{3}\right)$

(3R)-(+)-Methyl-N-(4'-fluorophenyl)succinimide (6). White solid. Mp: 105-107 ${ }^{\circ} \mathrm{C}$. Yield: $76 \%$. ${ }^{1} \mathrm{H}-\mathrm{NMR}$ $\left(\mathrm{CDCl}_{3}\right): 1.45\left(3 \mathrm{H}, \mathrm{d}, J=7.2 \mathrm{~Hz}, \mathrm{CH}_{3}\right) ; 2.50(1 \mathrm{H}, \mathrm{dd}, 17.1 ; 3.8 \mathrm{~Hz}, \mathrm{H}-3 \mathrm{~b}) ; 2.98-3.19(2 \mathrm{H}, \mathrm{m}, \mathrm{H}-2$ and $\mathrm{H}-3 \mathrm{a}) ; 7.15\left(2 \mathrm{H}, \mathrm{t}, J=8.7 \mathrm{~Hz}, \mathrm{H}-2^{\prime}, 6^{\prime}\right) ; 7.28\left(2 \mathrm{H}, \mathrm{dd}, J=8.0,5.8 \mathrm{~Hz}, \mathrm{H}-3^{\prime}, 5^{\prime}\right)$ ppm. ${ }^{13} \mathrm{C}^{-\mathrm{NMR}}\left(\mathrm{CDCl}_{3}\right)$ : $16.9 ; 34.8 ; 36.6 ; 116.2\left(\mathrm{~d}, J_{\mathrm{C}-\mathrm{F}}=22.9 \mathrm{~Hz}\right) ; 128.2(\mathrm{~d}, \mathrm{JC}-\mathrm{F}=8.8 \mathrm{~Hz}) ; 148.5 ; 162.3\left(\mathrm{~d}, J_{\mathrm{C}-\mathrm{F}}=248.7 \mathrm{~Hz}\right)$; 175.3; 179.4 ppm. MS: $m / z 207\left(\mathrm{M}^{+}, 100 \%\right), 250\left(\mathrm{M}^{+}+1,12 \%\right) .[\alpha]_{\mathrm{D}}^{27}+4.9 \pm 0.8\left(\mathrm{c} 0.72, \mathrm{CHCl}_{3}\right)$.

(3R)-(+)-Methyl-N-(4'-chlorophenyl)succinimide (7). White solid. Mp: $112-113{ }^{\circ} \mathrm{C}$. Yield: $68 \%$. ${ }^{1} \mathrm{H}-\mathrm{NMR}$ $\left(\mathrm{CDCl}_{3}\right): 1.46\left(3 \mathrm{H}, \mathrm{d}, J=7.2 \mathrm{~Hz}, \mathrm{CH}_{3}\right) ; 2.51(1 \mathrm{H}, \mathrm{dd}, J=17.1 ; 3.8 \mathrm{~Hz}, \mathrm{H}-3 \mathrm{~b}) ; 2.96-3.16(2 \mathrm{H}, \mathrm{m}, \mathrm{H}-2$ and $\mathrm{H}-3 \mathrm{a}) ; 7.26\left(2 \mathrm{H}, \mathrm{d}, J=8.8 \mathrm{~Hz}, \mathrm{H}-3^{\prime}, 5^{\prime}\right) ; 7.44\left(2 \mathrm{H}, \mathrm{d}, J=8.7 \mathrm{~Hz}, \mathrm{H}-2^{\prime}, 6^{\prime}\right)$ ppm. ${ }^{13} \mathrm{C}-\mathrm{NMR}\left(\mathrm{CDCl}_{3}\right)$ : $16.9 ; 34.8 ; 36.6 ; 126.2 ; 129.1 ; 136.4 ; 142.3 ; 178.4 ; 178.7$ ppm. MS: $m / z 223\left(\mathrm{M}^{+}, 100 \%\right), 225\left(\mathrm{M}^{+}+2\right.$, $32 \%), 224\left(\mathrm{M}^{+}+1,12 \%\right) .[\alpha]_{\mathrm{D}}^{27}+4.5 \pm 1.1\left(\mathrm{c} 0.66, \mathrm{CHCl}_{3}\right) .[\alpha]_{\mathrm{D}}^{27}+4.5 \pm 1.1\left(\mathrm{c} 0.66, \mathrm{CHCl}_{3}\right)$.

(3R)-(+)-Methyl-N-(4'-bromophenyl)succinimide (8). White solid. Mp: $134-136{ }^{\circ} \mathrm{C}$. Yield: $49 \%$. ${ }^{1} \mathrm{H}-\mathrm{NMR}$ $\left(\mathrm{CDCl}_{3}\right): 1.46\left(3 \mathrm{H}, \mathrm{d}, J=7.2 \mathrm{~Hz}, \mathrm{CH}_{3}\right) ; 2.51(1 \mathrm{H}, \mathrm{dd}, 17.1 ; 3.8 \mathrm{~Hz}, \mathrm{H}-3 \mathrm{~b}) ; 2.96-3.16(2 \mathrm{H}, \mathrm{m}, \mathrm{H}-2$ and H-3a); 7.19 (2H, d, $\left.J=8.8 \mathrm{~Hz}, \mathrm{H}-3^{\prime}, 5^{\prime}\right) ; 7.56\left(2 \mathrm{H}, \mathrm{d}, J=8.7 \mathrm{~Hz}, \mathrm{H}-2^{\prime}, 6^{\prime}\right)$ ppm. ${ }^{13} \mathrm{C}-\mathrm{NMR}\left(\mathrm{CDCl}_{3}\right)$ : $16.8 ; 34.9 ; 36.6 ; 122.4 ; 127.9 ; 130.9 ; 132.3 ; 174.8 ; 179.2$ ppm. MS: $m / z 267\left(\mathrm{M}^{+}, 100 \%\right), 269\left(\mathrm{M}^{+}+2\right.$, $98 \%), 268\left(\mathrm{M}^{+}+1,12 \%\right) .[\alpha]_{\mathrm{D}}^{27}+5.6 \pm 1.3\left(\mathrm{c} 0.58, \mathrm{CHCl}_{3}\right)$.

(3R)-(+)-Methyl-(2'-methylphenyl)succinimide (9). White solid. Mp: $102-105{ }^{\circ} \mathrm{C}$. Yield: $74 \% .{ }^{1} \mathrm{H}-\mathrm{NMR}$ $\left(\mathrm{CDCl}_{3}\right): 1.46\left(3 \mathrm{H}, \mathrm{dd}, J=7.15,1.46 \mathrm{~Hz}, \mathrm{CH}_{3}\right) ; 2.13\left(3 \mathrm{H}, \mathrm{d}, J=1.87 \mathrm{~Hz}, \mathrm{CH}_{3}\right) ; 2.52(1 \mathrm{H}$, ddd, $J=16.7,3.4,1.6 \mathrm{~Hz}, \mathrm{H}-3 \mathrm{~b}) ; 2.96-3.17$ (2H, m, H- 2;3a); 7.05 (1H, dd, $\left.J=7.2,1.1 \mathrm{~Hz} ; \mathrm{H}-4{ }^{\prime}\right)$; 7.24-7.38 (3H, m, H-3'; '; 6') ppm. ${ }^{13} \mathrm{C}-\mathrm{NMR}\left(\mathrm{CDCl}_{3}\right)$ : 16.9; 17.5; 35.2; 36.8; 126.9; 127.9; 129.5; $131.0 ; 131.2 ; 135.5 ; 175.4$; i179.6 ppm. MS: $m / z 201\left(\mathrm{M}^{+}, 100 \%\right), 202\left(\mathrm{M}^{+}+1,13 \%\right) .[\alpha]_{\mathrm{D}}^{27}+7.3 \pm 1.7$ (c $0.47, \mathrm{CHCl}_{3}$ )

(3R)-(+)-Methyl-N-(2',4'-difluorophenyl)succinimide (10). White solid. Mp: $102-104{ }^{\circ} \mathrm{C}$. Yield: $49 \%$. ${ }^{1} \mathrm{H}-\mathrm{NMR}\left(\mathrm{CDCl}_{3}\right): 1.45\left(3 \mathrm{H}, \mathrm{dd}, J=7.15,1.46 \mathrm{~Hz}, \mathrm{CH}_{3}\right) ; 2.52(1 \mathrm{H}, \mathrm{dd}, J=3.4,1.6 \mathrm{~Hz}, \mathrm{H}-3 \mathrm{~b})$; 2.95-3.16 (2H, m, H-2;3a); 6.79 (2H, q, $\left.J=9.3 \mathrm{~Hz} ; 5^{\prime}, 66^{\prime}-\mathrm{H}\right) ; 7.15\left(1 \mathrm{H}, \mathrm{dt}, J=8.6 \mathrm{~Hz} ; 3^{\prime}-\mathrm{H}\right) \mathrm{ppm} .{ }^{13} \mathrm{C}-\mathrm{NMR}$ $\left(\mathrm{CDCl}_{3}\right): 16.8 ; 35.2 ; 36.7 ; 105.3(\mathrm{dd}, J=26.4,23.5 \mathrm{~Hz}), 112.0(\mathrm{dd}, J=22.6,3.6 \mathrm{~Hz}), 116.08(\mathrm{dd}$, $J=13.4,3.9 \mathrm{~Hz}), 130.24(\mathrm{dd}, J=10.2,1.7 \mathrm{~Hz}), 157.7(\mathrm{dd}, J=255.1,12.8 \mathrm{~Hz}), 163.1(\mathrm{dd}, J=251.9$,

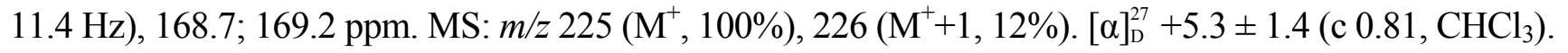




\subsection{Biotransformations}

A. fumigatus ATCC 26934 was grown on a plate with agarized Czapek culture medium for 3 days at $30{ }^{\circ} \mathrm{C}$ until well sporulated. 2-L Erlenmeyer flasks containing Czapek broth medium $(1 \mathrm{~L})$ were inoculated with suspensions of conidia $\left(2-5 \times 10^{6} \mathrm{CFU} \mathrm{mL}{ }^{-1}\right)$ and incubated at $30{ }^{\circ} \mathrm{C}$ for $72 \mathrm{~h}$ at $150 \mathrm{rpm}$ on an orbital shaker (Innova 4000, New Brunswick Scientific Inc., Edison, NJ, USA).

Substrates $(125 \mathrm{mg})$ in DMSO $(5 \mathrm{~mL})$ were poured into flasks containing the fungal biomass and the reaction mixtures were incubated at $30^{\circ} \mathrm{C}$ for $72 \mathrm{~h}$ on an orbital shaker $(150 \mathrm{rpm})$. Culture controls consisted of microorganism blank in which A. fumigatus ATCC 26934 was grown without substrate but fed with the same amount of DMSO. Substrate controls contained the sterile medium with the same amount of substrate and were incubated under the above conditions.

After incubation the mixtures were filtered, the aqueous phases were combined and extracted with ethyl acetate $(3 \times 250 \mathrm{~mL})$ and the organic phases were dried over $\mathrm{Na}_{2} \mathrm{SO}_{4}$. Conversion percentages were determined by GC analysis of the crude extracts and determined by using the TIC (total ion current) with the following equation: \% conversion: product TIC/(product TIC + substrate TIC) $\times 100$. Compounds were purified by silica gel column chromatography using a mixture of hexane and ethyl acetate (9:1) as eluent.

\subsection{Antifungal Susceptibility Testing}

The test fungi belonged either to the American Type Culture Collection (ATCC, Rockville, MD, USA), or the Culture Collection of CEREMIC (Centro de Referencia en Micología-CCC, Facultad de Ciencias Bioquímicas y Farmacéuticas, Rosario, Argentina). Species of Candida genus: C. albicans ATCC 10231, C. tropicalis CCC 131-2000, C. krusei CCC 117-2000, C. glabrata CCC 115-2000, C. parapsilosis CCC 124-2000, C. lusitaniae CCC 134-2000; S. cerevisiae ATCC 9763; C. neoformans ATCC 32264, M. gypseum CCC 115-2000, T. rubrum CCC 113-2000 and T. mentagrophytes ATCC 9972 were used.

Strains were grown on Sabouraud-chloramphenicol agar slants at $30{ }^{\circ} \mathrm{C}$, maintained on slopes of Sabouraud-dextrose agar (SDA, Oxoid), and sub-cultured every 15 days to prevent pleomorphic transformations. Inocula were obtained according to reported procedures $[23,24]$ and adjusted to $1-5 \times 10^{3}$ colony forming units (CFU) $\mathrm{mL}^{-1}$.

The antifungal activity was determined by using broth microdilution techniques following the guidelines of the CLSI for yeasts and for filamentous fungi $[23,24]$. Compounds stock solutions were two-fold diluted from 250 to $0.24 \mu \mathrm{g} \mathrm{ml}^{-1}$ (final volume $=100 \mu \mathrm{L}$ ) and a final DMSO (Sigma) concentration $<1 \%$ in the culture media RPMI-1640 (Sigma) buffered to $\mathrm{pH} 7.0$ with MOPS (Sigma).

The test was performed in 96 wells-microplates and included: Test wells (TW), containing the compound solution and the inoculum of the fungus; Growth Control Well (GCW), that contains compound-free medium and inoculum; Blank Test Wells (BTW), with the compound, culture medium and sterile water instead of inoculum; Sterility Control Well (SCW) containing the compound diluted in culture medium and sterile water. Ketoconazole (Sigma), terbinafine (Sigma) and amphotericin B (Sigma), were used as standard controls. 
Plates were incubated in a moist, dark chamber $24 \mathrm{~h}$ for yeasts and $5 \mathrm{~d}$ for dermatophytes and the MIC (Minimum Inhibitory Concentration) was determined. For yeasts, microplates turbidity due to fungal growth were determined at $405 \mathrm{~nm}$ in a VERSA Max microplate reader (Molecular Devices, Sunnyvale, CA, USA) and the $\%$ of growth were calculated as follows: $\%$ of growth $=$ $\left[\left(\mathrm{OD}_{\mathrm{TW}}-\mathrm{OD}_{\mathrm{BTW}}\right) /\left(\mathrm{OD}_{\mathrm{GCW}}-\mathrm{OD}_{\mathrm{SCW}}\right) \times 100\right.$.

\subsection{Statistical Tests}

Multiple comparisons were performed by the non-parametric ANOVA, Kruskal-Wallis test followed by Dunn's multiple comparisons and Wilcoxon's signed rank test. The associations between MIC for the different groups were corroborated with the Score's test [25]. Individual comparisons between groups were performed with Student's t test. A value of $p<0.05$ was considered significant.

\section{Conclusions}

The antifungal behavior of eighteen enantiomerically pure $(3 R)$-methyl- and $(3 R, 4 R)$-dimethyl- $N$ phenyl-, $N$-phenylalkyl- or $N$-arylsuccinimides, obtained by a low-cost and environmentally friendly A. fumigatus-catalyzed reduction of maleimides, were compared to that of the corresponding racemic ones. Results showed that the biotransformation products displayed statistically significant higher antifungal activity against a panel of human opportunistic pathogenic fungi including yeasts as well as dermatophytes than racemic ones as demonstrated by different approaches. These findings can be highly useful for the development of chiral methylated succinimides as antifungal drugs by the pharmaceutical industry and in a broader sense, they open new avenues for the development of highly active drugs containing other 3- and 3,4-substituted chiral succinimides [26].

\section{Acknowledgments}

MS and SZ acknowledge the Agencia Nacional de Promoción Científica y Tecnológica de Argentina (ANPCyT, PICT 0049-2010 and PICT 0608-2010) and the National University of Rosario (BIO 258) for funds. We gratefully acknowledge Prof. Liliana Racca from Statistical Department of the National University of Rosario for the analysis of results.

\section{Conflict of Interest}

The authors declare no conflict of interest.

\section{References}

1. Groutas, W.C.; Brubaker, M.J.; Chong, L.S.; Venkataraman, R.; Huang, H.; Epp, J.B.; Kuang, R., Hoidal, J.R. Design, synthesis and biological evaluation of succinimide derivatives as potential mechanism-based inhibitors of human leukocyte elastase, cathepsin G and proteinase 3 . Bioorg. Med. Chem. 1995, 3, 375-381.

2. Curtin, M.L.; Garland, R.B.; Heyman, H.R.; Frey, R.R.; Michaelides, M.R.; Li, J.; Glaser, K.; Marcotte, P.; Davidsen, S.K. Succinimide hydroxamic acids as potent inhibitors of histone deacetylase (HDAC). Bioorg. Med. Chem. Lett. 2002, 12, 2919-2923. 
3. Ibnusaud, I.; Thomas, G. Biologically interesting chiral 3,4-disubstituted pyrrolidines from optically active hydroxycitric acid lactones. Tetrahedron Lett. 2003, 44, 1247-1249.

4. Isaka, M.; Rugseree, N.; Maithip, P.; Kongsaeree, P.; Prabpai, S.; Thebtaranonth, Y. Hirsutellones A-E, antimycobacterial alkaloids from the insect pathogenic fungus Hirsutella nivea BCC 2594. Tetrahedron 2005, 61, 5577-5583.

5. Uddin, J.; Ueda, K.; Siwu, E.R.; Kita, M.; Uemura, D. Cytotoxic labdane alkaloids from an ascidian Lissoclinum sp.: Isolation, structure elucidation, and structure-activity relationship. Bioorg. Med. Chem. 2006, 14, 6954-6961.

6. Chauhan, P.; Jasneet K.; Chimni, S. Asymmetric organocatalytic addition reactions of maleimides: A promising approach towards the synthesis of chiral succinimide derivatives. Chem. Asian J. 2013, 8, 328-346.

7. Fredenhagen, A.; Tamura, S.Y.; Kenny, P.T.; Komura, H.; Naya, Y.; Nakanishi, K.; Nishiyama, K.; Sugiura, M.; Kita, H. Andrimid, a new peptide antibiotic produced by an intracellular bacterial symbiont isolated from a brown planthopper. J. Am. Chem. Soc. 1987, 109, 4409-4411.

8. Ohshima, T.; Tadaoka, H.; Hori, K.; Sayo, N.; Mashima, K. Highly enantio- and s-trans C=C bond selective catalytic hydrogenation of cyclic enones: Alternative synthesis of $(-)$-menthol. Chem. Eur. J. 2008, 14, 2060-2066.

9. Borges, K.B.; Borges, W.D.; Durán-Patrón, R.; Pupo, M.T.; Bonato, P.S.; Collado, I.G. Stereoselective biotransformations using fungi as biocatalysts. Tetrahedron Asymmetry 2009, 20, 385-397.

10. Valadez-Blanco, R.; Livingston, A.G. Enantioselective whole-cell biotransformation of acetophenone to $S$-phenylethanol by Rhodotorula glutinis: Part I. Product formation kinetics and feeding strategies in aqueous media. Biochem. Eng. J. 2009, 46, 44-53.

11. Patel, R.N. Biocatalysis: Synthesis of key intermediates for development of pharmaceuticals. ACS Catal. 2011, 1, 1056-1074.

12. Kasprzyk-Hordern, B. Pharmacologically active compounds in the environment and their chirality Chem. Soc. Rev. 2010, 39, 4466-4503.

13. Sortino, M.; Cechinel Filho, V.; Zacchino, S. Highly enantioselective reduction of the C-C double bond of $N$-phenyl-2-methyl-and $N$-phenyl-2,3-dimethyl-maleimides by fungal strains. Tetrahedron Asymmetry 2009, 20, 1106-1108.

14. Sortino, M.; Zacchino, S. Efficient asymmetric hydrogenation of the C-C double bond of 2-methyl-and 2,3-dimethyl- $N$-phenylalkylmaleimides by Aspergillus fumigatus. Tetrahedron Asymmetry 2010, 21, 535-539.

15. Hutt, A.G.; O'Grady, J. Drug chirality: A consideration of the significance of the stereochemistry of antimicrobial agents. J. Antimicrob. Chemother. 1996, 37, 7-32.

16. Sortino, M.; Zacchino, S. Highly enantiomeric hydrogenation of C-C double bound of methylated $N$-phenyl and $N$-phenylalkylmaleimides by Aspergillus fumigatus. In: Practical Methods for Biocatalysis and Biotransformations 2; Whittall, J., Sutton, P., Eds.; Wiley \& Sons: New York, NY, USA, 2012; pp. 108-114.

17. Balenović, K.; Bregant, N. Correlation of the configurations of $\alpha$-methyl- $\beta$-alanine and methylsuccinic acid. J. Chem. Soc. 1965, 5131-5132. 
18. Hegazy, M.E.; Shishido, K.; Hirata, T. Asymmetric hydrogenation of the C-C double bond of 1-and 1,2-methylated maleimides with cultured suspension cells of Marchantia polymorpha. Tetrahedron Asymmetry 2006, 17, 1859-1862.

19. Pfaller, M.A.; Diekema, D.J. Epidemiology of invasive candidiasis: A persistent public health problem. Clin. Microbiol. Rev. 2007, 20, 133-163.

20. Kontoyiannis, D.P.; Mantadakis, E.; Samonis, G. Systemic mycoses in the immunocompromised host: An update in antifungal therapy. J. Hosp. Inf. 2003, 53, 243-258.

21. Singh, N. Treatment of opportunistic mycoses: How long is long enough? Lancet Infect. Dis. 2003, 3, 703-708.

22. Weitzman, I.; Summerbell, R.C. The dermatophytes. Clin. Microbiol. Rev. 1995, 8, 240-259.

23. CLSI (Clinical and Laboratory Standards Institute) document. Reference Method for Broth Dilution Antifungal Susceptibility Testing of Yeasts; Approved Standard M27-A3; 3rd ed.; CLSI: Wayne, PA, USA, 2008; pp. 1-25.

24. CLSI (Clinical and Laboratory Standards Institute) document. Reference Method for Broth Dilution Antifungal Susceptibility Testing of Filamentous Fungi; Approved Standard M38-A2, 2nd ed.; CLSI: Wayne, PA, USA, 2008; pp. 1-35.

25. Stokes, M.; Davis, Ch.; Koch, G. Categorical Data Analysis Using the SAS System, 2nd ed.; SAS Institute: Cary, NC, USA. 2000.

26. Alcaide, B.; Almendros, P.; Cabrero, G.; Ruiz, M.P. Organocatalytic ring expansion of $\beta$-lactams to $\gamma$-lactams through a novel N1-C4 bond cleavage. Direct synthesis of enantiopure succinimide derivatives. Org. Lett. 2005, 7, 3981-3984.

Sample Availability: Samples of compounds 11-18 are available from the authors.

(C) 2013 by the authors; licensee MDPI, Basel, Switzerland. This article is an open access article distributed under the terms and conditions of the Creative Commons Attribution license (http://creativecommons.org/licenses/by/3.0/). 